\title{
THE MULTI-ATTRIBUTE GROUP DECISION MAKING METHOD BASED ON THE INTERVAL GREY LINGUISTIC VARIABLES WEIGHTED HARMONIC AGGREGATION OPERATORS
}

\author{
Fang JIN ${ }^{\mathrm{a}}$, Peide LIU $^{\mathrm{b}}$, Xin $\mathrm{ZHANG}^{\mathrm{c}}$ \\ ${ }^{a}$ The Mathematical and Economic Research Institute, Shandong University of Finance and Economics, \\ Jinan Shandong 250014, China \\ ${ }^{\mathrm{b}, \mathrm{c}}$ School of Management Science and Engineering, Shandong University of Finance and Economics, \\ Jinan Shandong 250014, China
}

Received 18 August 2011; accepted 28 January 2012

\begin{abstract}
With respect to the characteristics of fuzziness, complexity and uncertainty for many group-decision making problems in real world, the paper proposes a novel method based on the interval grey linguistic variables hybrid weighted harmonic aggregation operators to solve the multiple attribute group decision making problems in which the attribute values and the weights take the form of the interval grey linguistic variables. In the approach, the relative concepts and the operation rules of interval grey linguistic variables are defined, and some operators (such as interval grey linguistic weighted harmonic aggregation (IGLWHA) operator, interval grey linguistic ordered weighted harmonic aggregation (IGLOWHA) operator, and interval grey linguistic hybrid weighted harmonic aggregation (IGLHWHA) operator) are proposed to solve the group decision making problems. The computational results from an illustrative example have shown that the proposed approach is feasible and effective for the group-decision making problems.
\end{abstract}

Keywords: group decision-making; fuzzy numbers; grey fuzzy number; interval grey linguistic variables; the interval grey linguistic variables hybrid weighted harmonic aggregation operator.

Reference to this paper should be made as follows: Jin, F.; Liu, P.; Zhang, X. 2013. The multi-attribute group decision making method based on the interval grey linguistic variables weighted harmonic aggregation operators, Technological and Economic Development of Economy 19(3): 409-430.

JEL Classification: C02, C44, D81.

Corresponding author Peide Liu

E-mail: peide.liu@gmail.com 


\section{Introduction}

Multiple attribute decision making (MADM) has been extensively applied to various areas such as society, economics, management, military and engineering technology, etc. Since the objective things are complex, uncertainty and human thinking is ambiguous, the majority of multi-attribute decision-making problems are uncertain and fuzzy, so fuzziness is the major factor which should be considered in the process of decision making. On the other hand, decision-making problems have the greyness in the process of dealing with the incomplete information. "Greyness" means amount of information is smaller' and inadequate. For example, in agriculture planting, even if the sown area, seed, fertilizer, irrigation and other information are completely clear, it is still difficult to accurately predict the productive output. The productive output is grey. Another example, in 2050, China's total population will be controlled between 15 and 16 billion. This "between 15 and 16 billion" is a concept of grey, we cannot know the accurate value. So, the "greyness" is a concept about "quantity". However, "fuzziness" means a concept is not clear. For example, about "young people", it is very difficult to designate an exact range in which they are young people and out which they are not, so it is fuzzy. Other examples, such as "hot water", "wet" etc. are fuzzy. So "fuzziness" is a concept about "quality". Obviously, "greyness" and "fuzziness" don't mean that some information is "grey information" and the other part of the information is "fuzzy information" for the same problem because they don't describe the same concept (Bu, Zhang 2002). In general, we can simply interpret "greyness" and "fuzziness" as width and depth of an evaluation object. In reality, the decision making problems have not only the fuzziness, but also the greyness, which are called the grey fuzzy multi-attribute decision making problems. For example, about ability of innovation management of enterprises, it has the fuzziness and greyness simultaneously, because the concept of ability of innovation management is unclear, i.e., it has the fuzziness; at the same time, we cannot get all information about ability of innovation management of enterprises, so it has the greyness. There are similar examples, such as moral evaluation, working ability assessment, evaluation of a person's level of knowledge, etc.

About fuzzy theory, Zadeh (1965) firstly proposed the theory of Fuzzy Sets, The core idea is to extend membership function to any value in the closed interval $[0,1]$. Then fuzzy sets had been extended to interval numbers, triangular fuzzy numbers, trapezoidal fuzzy numbers, linguistic variables, intuitionistic fuzzy numbers, etc. and widely used in the field of decision-making (Herrera et al. 1996; Liu 2011; Liu, Su 2010; Liu, Zhang 2010; Zhang, Liu 2010; Yu 2013; Razavi Hajiagha et al. 2013). About grey theory, Deng (1982) firstly proposed the theory of Grey Systems, then grey theory has been the rapid development, and a series of grey decision-making methods were proposed (Deng 2002; Liu, W. L., Liu, P. D. 2010). The grey fuzzy theory, which combined the fuzzy theory and the grey theory, takes into account the greyness and fuzzynes of decision making problems, and is more in line with the objective reality of things. At present, there have been consistent efforts to the research on grey fuzzy decision making problems. Bu and Zhang (2002), Choobineh and Li (1993a, b), Jin and Lou (2003, 2004), Luo and Liu (2004) studied the ranking method of grey fuzzy number. Bu and Zhang (2002) transformed the grey fuzzy number into the interval number, and then utilized the ranking method of interval number to rank the order 
of alternatives. For the grey fuzzy multiple attribute decision making problems which both the fuzzy part and the grey part took the form of real number, Jin and Lou (2003) proposed the decision making model which utilized the difference between the alternatives and the fuzzy positive ideal solution, and between the alternatives and the negative ideal solution to rank the orders based on the Hamming distance. Jin and Lou (2004) utilized the distance between each alternative and the grey fuzzy ideal solution to rank the orders of alternatives. In order to solve the grey fuzzy decision making problems, Luo and Liu (2004) utilized the maximum entropy formula to determine attribute weights, then ranked the orders of alternatives based on the linear combination of fuzzy information and grey information. Zhu et al. (2006) constructed the evaluation model in which the fuzzy part and the grey part took the form of interval number and the real number respectively. Meng et al. (2007) proposed the interval numbers to present greyness and fuzziness of grey fuzzy decision making problems, and the mathematical model of interval valued grey fuzzy comprehensive evaluation is established, and the application to the selection of the preferred project is given. Wang and Wang (2008) extended the fuzzy part and the grey part of grey fuzzy decision making problems to interval numbers, and ranked the order of alternatives based on the ordered weight aggregation (OWA) operator. Zhang (2013) proposed the interval grey linguistic variables ordered weighted aggregation (IGLOWA) operator, and then use the Choquet integral to develop the interval grey linguistic correlated ordered arithmetic aggregation (IGLCOA) operator and the interval grey linguistic correlated ordered geometric aggregation (IGLCOGA) operator.

Because the linguistic variables are easier to express fuzzy information, and the research on multi-attribute decision making based on the linguistic variables has made great achievements (Alonso et al. 2009; Cabrerizo et al. 2010a, b; Herrera et al. 2009; Herrera-Viedma et al. 2003; Kim, Ahn 1999; Martínez et al. 2009; Xu 2004, 2007, 2008). So, this paper proposes the concept of interval grey linguistic variables in which the fuzzy part and the grey part adopt linguistic variables and interval numbers respectively, and then studies the operation rules and the multiple attribute decision making method based on interval grey linguistic variables.

The remainder of the paper is organized as follows: Section 1 introduces some relative knowledge; Section 2 defines the interval grey linguistic variables and proposes some weighted harmonic aggregation operators; Section 3 gives a method based on the interval grey linguistic variables hybrid weighted harmonic aggregation operators to solve the multiple attribute group decision making problems; Section 4 presents an illustrative example to verify effectiveness of this method and to illustrate its decision making steps; Finally, conclusions are given in the final section.

\section{Preliminaries}

\subsection{Grey number (Deng 2002; Luo 2005; Lu 2009)}

Grey number is the basic unit to express the greyness. We can call only knowing the ranges roughly and not knowing the exact value as grey number. In the application, the grey number 
generally refers to a range or an uncertain number, and it can be expressed by “ $\otimes$ ”. Grey number can be divided into the following categories:

1) The grey number only with a lower bound

The grey number in this type can be expressed as $\mu_{A}(x) \rightarrow[0,1]$, where $\underline{a}$ is the lower bound of the grey number $\otimes$ and it's also a certain number.

2) The grey number only with a upper bound

The grey number in this type can be expressed as $\otimes \in(-\infty, \bar{a}]$, where $\bar{a}$ is the upper bound of the grey number $\otimes$ and it's also a certain number.

3) The grey number with interval number

The grey number in this type can be expressed as $\otimes \in[\underline{a}, \bar{a}]$, where $\underline{a}$ and $\bar{a}$ are a certain number, and $\underline{a}$ is the lower bound, $\bar{a}$ is the upper bound of the grey number $\otimes$.

4) The grey number with three-point interval number

The grey number in this type can be expressed as $\otimes \in[\underline{a}, a, \bar{a}]$, where $\underline{a}, a$ and $\bar{a}$ are a certain number; $\underline{a}$ and $\nabla$ iare the lower bound and upper bound of the grey number $\otimes$ respectively, and $a$ is the center of gravity which can be get most likely.

5) The black number and white number

When grey number $\otimes \in(-\infty,+\infty)$, we can call $\otimes$ as a black number. It shows that information is completely unknown; when grey number $\otimes \in[\underline{a}, \bar{a}]$ and $\underline{a}=\bar{a}$, we can call $\otimes$ as a white number. It shows information is completely known.

\subsection{Grey fuzzy math}

\section{(Chen 1994; Li and Wang 1994; Wang and Song 1988; Wang 1996)}

Definition 1: Let $\underset{\otimes}{\tilde{A}}$ be the fuzzy subset in the space $X=\{x\}$, if the membership degree $\mu_{A}(x)$ of $x$ to $\underset{\otimes}{\tilde{A}}$ has the greyness $v_{A}(x)$ in the interval $[0,1]$, then $\underset{\otimes}{\tilde{A}}$ is called the grey fuzzy set in space $X$ :

$$
\underset{\Theta}{\tilde{A}}=\left\{\left(x, \mu_{A}(x), v_{A}(x)\right) \mid x \in X\right\} .
$$

The set pair mode is $\underset{\otimes}{\tilde{A}}=(\tilde{A}, A)$, where $\tilde{A}=\left\{\left(x, \mu_{A}(x)\right) \mid x \in X\right\}$ is called the fuzzy part of $\tilde{A}$, and $\underset{\otimes}{A}=\left\{\left(x, v_{A}(x)\right) \mid x \in X\right\}$ is called the grey part of $\underset{\otimes}{\tilde{A}}$.

So the grey fuzzy set is regarded as the generalization of the fuzzy set and the grey set.

Definition 2: Let $X=\{x\}$ and $Y=\{y\}$ be the given space, if $v_{R}(x, y)$ is the greyness of the membership function $\mu_{R}(x, y)$ of $\tilde{R}$ which is the fuzzy relationship between $x$ and $y$, then grey fuzzy set $\underset{\otimes}{\tilde{R}}=\left\{\left((x, y), \mu_{R}(x, y), v_{R}(x, y)\right) \mid x \in X, y \in Y\right\}$ is called the grey fuzzy relationship in direct product space $X \times Y$, which is represented as the grey fuzzy matrix mode:

$$
\tilde{R}=\left[\begin{array}{cccc}
\left(\mu_{11}, v_{11}\right) & \left(\mu_{12}, v_{12}\right) & \cdots & \left(\mu_{1 n}, v_{1 n}\right) \\
\left(\mu_{21}, v_{21}\right) & \left(\mu_{22}, v_{22}\right) & \ldots & \left(\mu_{2 n}, v_{2 n}\right) \\
\vdots & \vdots & \vdots & \vdots \\
\left(\mu_{m 1}, v_{m 1}\right) & \left(\mu_{m 2}, v_{m 2}\right) & \cdots & \left(\mu_{m n}, v_{m n}\right)
\end{array}\right] .
$$


And $\underset{\otimes}{\tilde{R}}=(\underset{R}{\tilde{R}, R} \underset{\otimes}{ })$ represents the grey fuzzy relationship in direct product space $X \times Y$, where $\tilde{R}=\left\{\left((x, y), \mu_{A}(x, y)\right) \mid x \in X, y \in Y\right\}$ represents the fuzzy relationship in direct product space $X \times Y$, and $R=\left\{\left((x, y), v_{A}(x, y)\right) \mid x \in X, y \in Y\right\}$ represents the grey relationship in direct product space $X \times Y$.

\subsection{Linguistic evaluation set and its extension}

Suppose that $S=\left(s_{1}, s_{2}, \cdots, s_{l}\right)$ is a finite and totally ordered discrete term set, where $l$ is the odd number. In real situation, $l$ is equal to $3,5,7,9$ etc. In this paper, $l=7$. For example, a set $S$ could be given as follows:

$S=\left(s_{1}, s_{2}, s_{3}, s_{4}, s_{5}, s_{6}, s_{7}\right)=\{$ very poor, poor, slightly poor, fair, slightly good, good, very good $\}$.

Usually, in these cases, it requires that $s_{i}$ and $s_{j}$ must satisfy the following additional characteristics (Herrera, Herrera-Viedma 2000):

1) The set is ordered: $s_{i} \prec s_{j}$, if and only if $i<j$;

2) There is the negation operator: $\operatorname{neg}\left(s_{i}\right)=s_{l-i}$;

3) Maximum operator: $\max \left(s_{i}, s_{j}\right)=s_{i}$, if $i \geq j$;

4) Minimum operator: $\min \left(s_{i}, s_{j}\right)=s_{i}$, if $i \leq j$.

For any linguistic labels $S=\left(s_{1}, s_{2}, \cdots, s_{l}\right)$, the relationship between the element $s_{i}$ and its subscript $i$ is strictly monotone increasing (Herrera et al. 1996; Xu 2006a), so the function can be defined as follows:

$$
f: s_{i}=f(i)
$$

Obviously, the function $f(i)$ is the strictly monotone increasing function about subscript $i$. To preserve all the given information, the discrete linguistic label $S=\left(s_{1}, s_{2}, \cdots, s_{l}\right)$ is extended to a continuous linguistic label $\bar{S}=\left\{s_{\alpha} \mid \alpha \in R\right\}$ which satisfied the above characteristics. If $s_{\alpha} \in S$, then $s_{\alpha}$ is called an original linguistic label, otherwise, $s_{\alpha}$ is called a virtual linguistic label. In general, the decision maker uses the original label to evaluate attributes and alternatives, and the virtual labels can only appear in the course of operation.

Let $s_{i}, s_{j} \in \bar{S}$ and $\lambda_{1}, \lambda_{2} \in[0,1], n$ is a positive integer, then operational laws of linguistic variables are given as follows (Xu 2006b):

1) $\beta s_{i}=s_{\beta \times i}$;

2) $s_{i} \oplus s_{j}=s_{i+j}$;

3) $s_{i} / s_{j}=s_{i / j}$, if $j \neq 0$;

4) $\left(s_{i}\right)^{n}=s_{i^{n}}$;

5) $\lambda_{1}\left(s_{i} \oplus s_{j}\right)=\lambda_{1} s_{i} \oplus \lambda_{1} s_{j},\left(\lambda_{1}+\lambda_{2}\right) s_{i}=\lambda_{1} s_{i} \oplus \lambda_{2} s_{i}$. 
Definition 3: Let $s_{\alpha}, s_{\beta}$ be the two linguistic variables, then the distance between $s_{\alpha}$ and $s_{\beta}$ is defined as follows:

$$
d\left(s_{\alpha}, s_{\beta}\right)=|\alpha-\beta| / l
$$

\section{Interval grey linguistic variables}

\subsection{The definition of interval grey linguistic variables}

Definition 4: Let $\tilde{A}=(\tilde{A}, \underset{\otimes}{A})$ be the grey fuzzy number, if its fuzzy part $\tilde{A}$ is a linguistic variable $s_{\alpha} \in \bar{S}$, and its grey part $A_{\otimes}$ is a closed interval $\left[g_{A}^{L}, g_{A}^{U}\right] \subseteq[0,1]$, where $g_{A}^{L}$ is the interval lower limit, $g_{A}^{U}$ is the interval upper limit, and $g_{A}^{L} \leq g_{A}^{U}$, then $\underset{\otimes}{\tilde{A}}$ is called the interval grey linguistic variables.

Because the linguistic variables are easier to express fuzzy information, it is more reasonable to utilize the linguistic variables to represent the fuzzy part, and for the grey part which indicates the amount of information obtained, it is more accurate to reflect the information obtained by decision maker using the interval numbers. The larger the greyness of the grey part is, the less information obtained and the lower credibility of the obtained information is. The lower the credibility of the obtained value is, the lower the usage value of the information is. When the greyness rises, the obtained information becomes useless. On the other hand, the smaller the greyness is, the more information obtained is, which causes higher credibility of the obtained value. That finally leads to higher usage value of the obtained information.

\subsection{The operation of the interval grey linguistic variables}

Supposed that $\underset{\otimes}{\tilde{A}}=\left(s_{\alpha},\left[g_{A}^{L}, g_{A}^{U}\right]\right), \underset{\otimes}{\tilde{B}}=\left(s_{\beta},\left[g_{B}^{L}, g_{B}^{U}\right]\right)$ and $\underset{\otimes}{\tilde{C}}=\left(s_{\lambda},\left[g_{C}^{L}, g_{C}^{U}\right]\right)$ are the three interval grey linguistic variables. Based on the concept of the interval grey linguistic variables, the linguistic operational rules and extension principle, the operation rules of interval grey linguistic variables are defined as follows:
1) $\underset{\otimes}{\tilde{A}}+\underset{\otimes}{\tilde{B}}=\left(s_{\alpha+\beta},\left[\max \left(g_{A}^{L}, g_{B}^{L}\right), \max \left(g_{A}^{U}, g_{B}^{U}\right)\right]\right)$;
2) $\underset{\otimes}{\tilde{A}}-\underset{\otimes}{\mathbb{B}}=\left(s_{\alpha-\beta},\left[\max \left(g_{A}^{L}, g_{B}^{L}\right), \max \left(g_{A}^{U}, g_{B}^{U}\right)\right]\right)$;
3) $\underset{\otimes}{\tilde{A}} \times \underset{\otimes}{\tilde{B}}=\left(s_{\alpha \times \beta},\left[\max \left(g_{A}^{L}, g_{B}^{L}\right), \max \left(g_{A}^{U}, g_{B}^{U}\right)\right]\right)$;
4) $\underset{\otimes}{\tilde{A}} / \underset{\otimes}{\tilde{B}}=\left(s_{\alpha / \beta},\left[\max \left(g_{A}^{L}, g_{B}^{L}\right), \max \left(g_{A}^{U}, g_{B}^{U}\right)\right]\right)$;
5) $\underset{\otimes}{\tilde{A}}=\left(s_{k \times \alpha},\left[g_{A}^{L}, g_{A}^{U}\right]\right)$;
6) $\left(\begin{array}{c}\tilde{A} \\ \otimes\end{array}\right)^{k}=\left(s_{\alpha^{k}},\left[g_{A}^{L}, g_{A}^{U}\right]\right)$. 


\subsection{The distance between the two interval grey linguistic variables}

Definition 5: Let $\underset{\otimes}{\tilde{A}}, \underset{\otimes}{\tilde{B}}, \underset{\otimes}{\tilde{C}}$ be the interval grey linguistic variables, $\underset{\otimes}{\tilde{Z}}$ be the set of the interval grey linguistic variables, $f$ be the mapping, $f: \underset{\otimes}{\tilde{Z}} \times \underset{\otimes}{\tilde{Z}} \rightarrow \underset{\otimes}{\tilde{R}}$.If $d(\underset{\otimes}{\tilde{A}}, \tilde{B})$ satisfies the following equation:

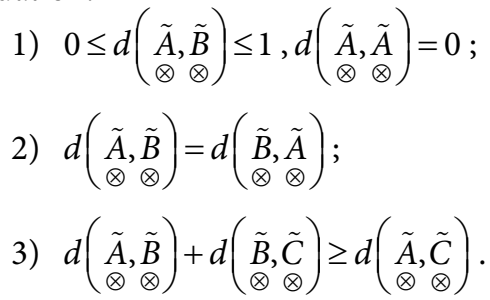

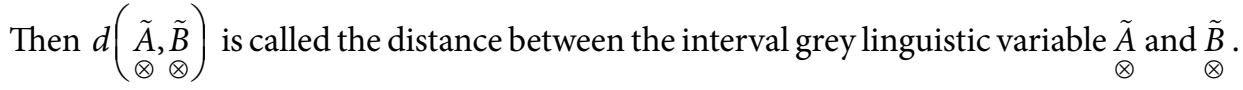
Definition 6: Let $\underset{\otimes}{\tilde{A}}=\left(s_{\alpha},\left[g_{A}^{L}, g_{A}^{U}\right]\right)$ and $\underset{\otimes}{\tilde{B}}=\left(s_{\beta},\left[g_{B}^{L}, g_{B}^{U}\right]\right)$ be the interval grey linguistic variables, then the Hamming distance $d(\underset{\otimes}{\tilde{A}, \tilde{B}})$ between the interval grey linguistic variable $\underset{\otimes}{\tilde{A}}$ and $\underset{\otimes}{\tilde{B}}$ is defined as follows:

$$
d(\underset{\otimes}{\tilde{A}, \tilde{B}})=\frac{1}{2(l-1)}\left(\left|\alpha\left(1-g_{A}^{L}\right)-\beta\left(1-g_{B}^{L}\right)\right|+\left|\alpha\left(1-g_{A}^{U}\right)-\beta\left(1-g_{B}^{U}\right)\right|\right) .
$$

It is easy to verify that the equation (15) satisfies the three equation of definition 5.

Specially, if $g_{A}^{L}=g_{A}^{U}=g_{B}^{L}=g_{B}^{U}=0$, then the interval grey linguistic variable is reduced to linguistic variable, and the equation (15) is transformed into equation (8). That is, the equation (8) is the special case of equation (15).

\subsection{The comparing method of interval grey linguistic variables}

\section{(1) C-OWA aggregate operator}

Definition 7 (Yager 2004): function $\rho:[0,1] \rightarrow[0,1]$ satisfied that:

1) $\rho(0)=0$;

2) $\rho(1)=1$;

3) if $x>y$, then $\rho(x)>\rho(y)$,

then $\rho$ is called the basic unit-interval monotonic (BUM) function.

Definition 8 (Yager 2004): Let $[a, b]$ be the interval number, and

$$
f_{\rho}([a, b])=\int_{0}^{1} \frac{d \rho(y)}{d y}(b-y(b-a)) d y,
$$

then $f$ is called the continuous interval number OWA (C-OWA)operator.

$$
\text { If } \rho(y)=y^{\delta}(\delta \geq 0) \text {, then } f_{\rho}([a, b])=\frac{b+\delta a}{\delta+1} \text {. }
$$


(2) The expectation value and the rank method of interval grey linguistic variables

Let $\underset{\otimes}{\tilde{A}}=\left(s_{\alpha},\left[g_{A}^{L}, g_{A}^{U}\right]\right)$ be the interval linguistic variable, the expectation value of interval grey linguistic variable $\underset{\otimes}{\tilde{A}}$ is defined as follows:

$$
I(\underset{\Theta}{\tilde{A}})=s_{\alpha} \times f_{\rho}\left(\left[\left(1-g_{A}^{U}\right),\left(1-g_{A}^{L}\right)\right]\right) .
$$

Suppose $\underset{\otimes}{\tilde{A}}=\left(s_{\alpha},\left[g_{A}^{L}, g_{A}^{U}\right]\right)$ and $\underset{\otimes}{\tilde{B}}=\left(s_{\beta},\left[g_{B}^{L}, g_{B}^{U}\right]\right)$ are two interval linguistic variables, if $I(\underset{\Theta}{\tilde{A}})>I(\underset{\otimes}{\tilde{B}})$, then $\underset{\otimes}{\tilde{A}}>\underset{\otimes}{\tilde{B}}$, and If $I \underset{\otimes}{\tilde{A}})=I \underset{\otimes}{\tilde{B}})$ and $s_{\alpha} \succ s_{\beta}$, then $\underset{\otimes}{\tilde{A}}>\underset{\otimes}{\tilde{B}}$.

\subsection{Interval grey linguistic variables hybrid weighted harmonic aggregation (IGLHWHA) operator}

Definition 9: Let $\underset{\otimes_{j}}{\tilde{A}}=\left(s_{\alpha_{j}},\left[g_{j}^{L}, g_{j}^{U}\right]\right)$ be the group of interval grey linguistic variables, and IGLWHA: $\Omega^{n} \rightarrow \Omega$, if

$$
\operatorname{IGLWHA} A_{W}\left(\underset{\otimes_{1}}{\tilde{A}, \tilde{A},}, \cdots, \underset{\otimes_{n}}{\tilde{A}}\right)=\left(\sum_{j=1}^{n} \frac{w_{j}}{\tilde{A}}\right)^{-1},
$$

where $\Omega$ is the set of all the interval grey linguistic variables, $W=\left(w_{1}, w_{2}, \cdots, w_{n}\right)$ is the weight vector of $\underset{\otimes}{\tilde{A}}(j=1,2, \cdots, n)$, and $\sum_{j=1}^{n} w_{j}=1$, then IGLWHA is called the interval grey linguistic weighted harmonic aggregation (IGLWHA)operator.

Based on the operation rules of interval grey linguistic variables, the equation (18) is deduced to:

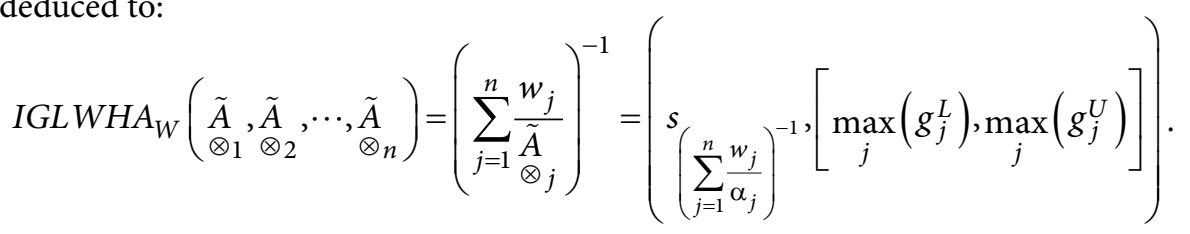

Example 1. Assume $W=(0.2,0.3,0.1,0.4)$ and

$$
\underset{\otimes_{1}}{\tilde{A}}=\left(s_{2},[0.2,0.4]\right), \underset{\otimes_{2}}{\tilde{A}}=\left(s_{1},[0.1,0.3]\right), \underset{\otimes_{3}}{\tilde{A}}=\left(s_{5},[0.4,0.5]\right), \underset{\otimes_{4}}{\tilde{A}}=\left(s_{4},[0.3,0.6]\right) .
$$


By Definition 9, we have

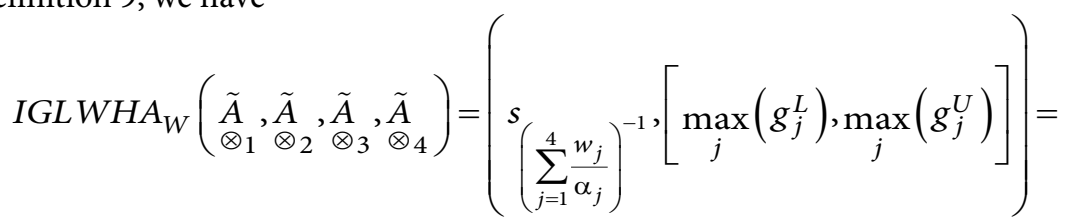

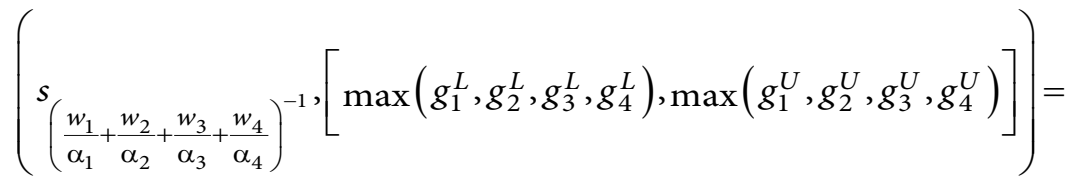

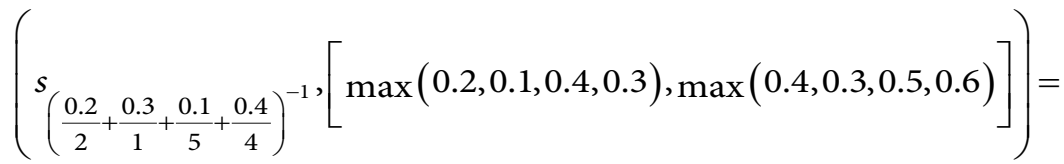

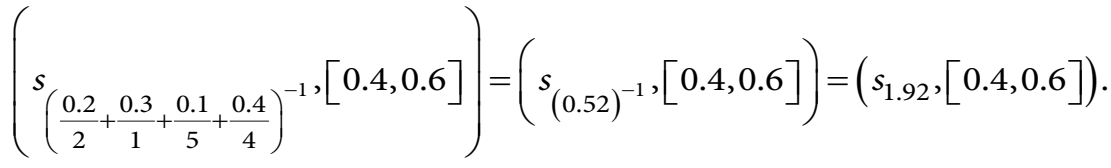

It's easy to prove that the IGLWHA operator has the following properties.

1) Theorem 1 (Commutativity)

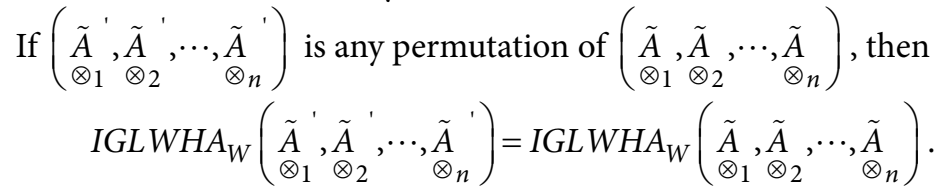

2) Theorem 2 (Idempotency)

If $\underset{\otimes}{A}=\tilde{A}$ for all $j$, then

$$
I G L W H A_{W}\left(\begin{array}{c}
\tilde{A}, \tilde{A} \\
\otimes_{1} \otimes_{2}, \cdots, \underset{\otimes_{n}}{\tilde{A}}
\end{array}\right)=\underset{\otimes}{\tilde{A}} .
$$

3) Theorem 3 (Monotonicity)

If $\underset{\otimes_{j}}{\tilde{A}} \leq \underset{\otimes_{j}}{\tilde{A}^{\prime}}$ for all $j$, then

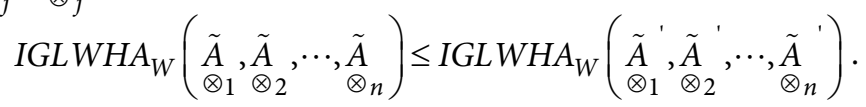

Definition 10: Let $\underset{\otimes_{j}}{\tilde{A}}=\left(s_{\alpha_{j}},\left[g_{j}^{L}, g_{j}^{U}\right]\right)$ be the group of interval grey linguistic variables, and IGLOWHA : $\Omega^{n} \rightarrow \Omega$, if

$$
\operatorname{IGLOWH} A_{\omega}\left(\underset{\otimes_{1}}{\tilde{A}, \tilde{A}}, \cdots, \underset{\otimes_{n}}{\tilde{A}}\right)=\left(\sum_{j=1}^{n} \frac{\omega_{j}}{\tilde{\otimes_{\sigma_{j}}}}\right)^{-1},
$$

where $\Omega$ is the set of all the interval grey linguistic variables, $\omega=\left(\omega_{1}, \omega_{2}, \cdots, \omega_{n}\right)$ is the weight vector associated with the function IGLOWHA and $\sum_{j=1}^{n} \omega_{j}=1$. Supposed that the 
possible permutation of $(1,2, \cdots, n)$ is $\left(\sigma_{1}, \sigma_{2}, \cdots, \sigma_{n}\right)$, and for any $j$, we can get $\underset{\otimes \sigma_{j-1}}{\tilde{A}} \geq \underset{\otimes \sigma_{j}}{\tilde{A}}$, then IGLOWHA is called the interval grey linguistic ordered weighted harmonic aggregation (IGLOWHA)operator.

Based on the operation rules of interval grey linguistic variables, the equation (20) is deduced to:

$$
\begin{aligned}
& \operatorname{IGLOWH} A_{\omega}\left(\underset{\otimes_{1}}{\tilde{A}}, \underset{\otimes_{2}}{\tilde{A}}, \cdots, \underset{\otimes_{n}}{\tilde{A}}\right)=\left(\sum_{j=1}^{n} \frac{\omega_{j}}{\tilde{A}}\right)^{-1}= \\
& \left.\left(\sum_{\sigma_{j}}\right)^{n} \frac{w_{j}}{\alpha_{\sigma_{j}}}\right)^{-1},\left[\max _{j}\left(g_{j}^{L}\right), \max _{j}\left(g_{j}^{U}\right)\right] .
\end{aligned}
$$

The characteristic of IGLOWHA operator is that interval grey linguistic variables $\tilde{A}(j=1,2, \cdots, n)$ is ranked in descending order and aggregated with weights. $\omega_{j}$ is associated with the $j^{\text {th }}$ position of the aggregation process, and $\underset{\otimes}{\tilde{A}}$ isn't associated with $\omega_{j}$. So $\omega$ is called the position weighted vector.

According to the actual situation, the position weight vector $\omega=\left(\omega_{1}, \omega_{2}, \cdots, \omega_{n}\right)$ is determined by the following method:

1) The method by Wang and $\mathrm{Xu}(2008)$ :

$$
\omega_{1}=\frac{1-\alpha}{n}+\alpha, \omega_{j}=\frac{1-\alpha}{n}, j \neq 1, \alpha \in[0,1] .
$$

2) The position weighted vector $\omega$ is determined by the method which proposed by Wang and $\mathrm{Xu}$ (2008). The equation is shown as follows:

$$
\omega_{i+1}=\frac{C_{n-1}^{i}}{2^{n-1}} \quad i=0,1, \cdots, n-1 .
$$

Example 2. Assume $\omega=\left(\frac{1}{8}, \frac{3}{8}, \frac{3}{8}, \frac{1}{8}\right)$ (calculated by Eq. 23) and

$$
\underset{\otimes_{1}}{\tilde{A}}=\left(s_{2},[0.2,0.4]\right), \underset{\otimes_{2}}{\tilde{A}}=\left(s_{1},[0.1,0.3]\right), \underset{\otimes_{3}}{\tilde{A}}=\left(s_{5},[0.4,0.5]\right), \underset{\otimes_{4}}{\tilde{A}}=\left(s_{4},[0.3,0.6]\right) .
$$

Firstly, calculate the expectation value of interval grey linguistic variables $\tilde{A}, \tilde{A}, \tilde{A}$ and $\tilde{A}$ according to Eq. (17).

Suppose $\rho(y)=y^{2}$, then $f_{\rho}([a, b])=\frac{b+2 a}{3}$. So,

$$
\begin{aligned}
& \left.I \underset{\otimes_{1}}{(\tilde{A}}\right)=s_{\alpha_{1}} \times f_{\rho}\left(\left[\left(1-g_{1}^{U}\right),\left(1-g_{1}^{L}\right)\right]\right)=s_{2} \times f_{\rho}([(1-0.4),(1-0.2)])= \\
& s_{2} \times f_{\rho}([0.6,0.8])=s_{2} \times \frac{0.8+2 \times 0.6}{3}=s_{2} \times 0.67=s_{1.34} .
\end{aligned}
$$


Similarly,

$$
\begin{aligned}
& \left.I \underset{\otimes_{2}}{(\underset{A}{\tilde{A}})}\right)=s_{\alpha_{2}} \times f_{\rho}\left(\left[\left(1-g_{2}^{U}\right),\left(1-g_{2}^{L}\right)\right]\right)=s_{1} \times f_{\rho}([(1-0.3),(1-0.1)])=s_{1} \times \frac{0.9+2 \times 0.7}{3}=s_{0.77} ; \\
& \left.I \underset{\otimes_{3}}{(\tilde{A}}\right)=s_{\alpha_{3}} \times f_{\rho}\left(\left[\left(1-g_{3}^{U}\right),\left(1-g_{3}^{L}\right)\right]\right)=s_{5} \times f_{\rho}([(1-0.5),(1-0.4)])=s_{5} \times \frac{0.6+2 \times 0.5}{3}=s_{2.65} ; \\
& I\left(\underset{\otimes_{4}}{\tilde{A}}\right)=s_{\alpha_{4}} \times f_{\rho}\left(\left[\left(1-g_{4}^{U}\right),\left(1-g_{4}^{L}\right)\right]\right)=s_{4} \times f_{\rho}([(1-0.6),(1-0.3)])=s_{4} \times \frac{0.7+2 \times 0.4}{3}=s_{2.00} .
\end{aligned}
$$

Secondly, we rank the $\underset{\otimes_{1}}{\tilde{A}}, \underset{\otimes_{2}}{\tilde{A}}, \underset{\otimes_{3}}{\tilde{A}}$ and $\underset{\otimes_{4}}{\tilde{A}}$, the results are shown as follows:

$$
\sigma_{1}=3, \sigma_{2}=4, \sigma_{3}=1, \sigma_{4}=2 \text {. }
$$

Finally, by definition 10 , we have:

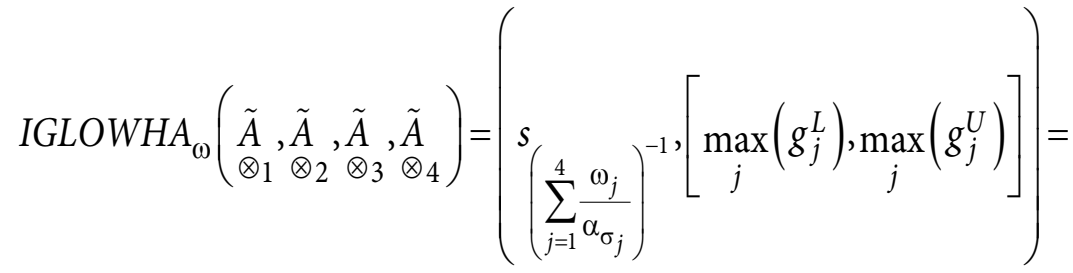

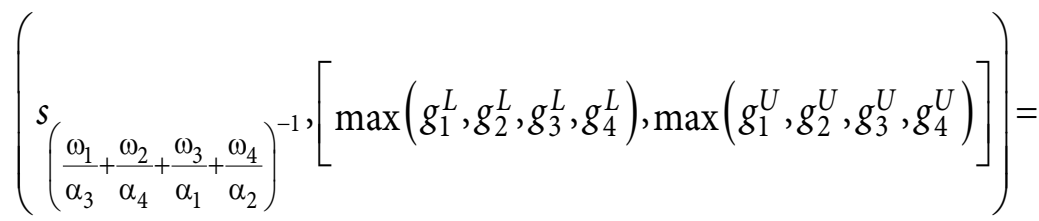

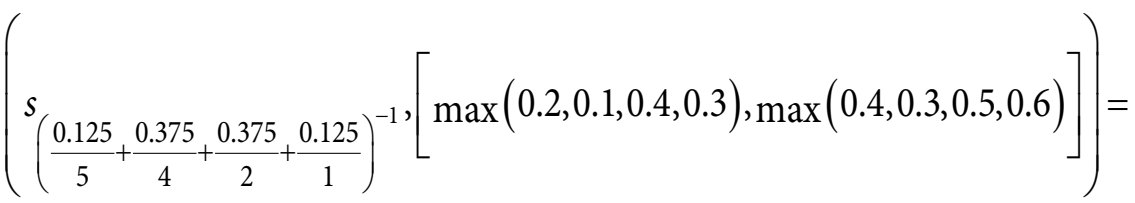

$$
\begin{aligned}
& \left(s_{(0.43)^{-1}},[0.4,0.6]\right)=\left(s_{2.32},[0.4,0.6]\right) \text {. }
\end{aligned}
$$

It's easy to prove that the IGLOWHA operator has the following properties.

1) Theorem 1 (Commutativity).

If $\left(\begin{array}{c}\tilde{A}_{\otimes_{1}}^{\prime}, \tilde{A}_{\otimes_{2}}^{\prime}, \cdots, \tilde{\otimes}_{\otimes_{n}}^{\prime} \\ \tilde{N}^{\prime}\end{array}\right)$ is any permutation of $\left(\begin{array}{c}\tilde{A}, \tilde{A}, \cdots, \tilde{A} \\ \otimes_{1} \otimes_{2}\end{array}\right)$, then

$$
\operatorname{IGLOWH} A_{\omega}\left(\underset{\tilde{\otimes}_{1}}{\tilde{A}^{\prime}, \tilde{\otimes}_{2}}{ }^{\prime}, \cdots, \tilde{\otimes}_{n}{ }^{\prime}\right)=\operatorname{IGLOWH} A_{\omega}\left(\underset{\otimes_{1}}{\tilde{A}}, \underset{\otimes_{2}}{\tilde{A}}, \cdots, \underset{\otimes_{n}}{\tilde{A}}\right) .
$$

\section{2) Theorem 2 (Idempotency)}

If $\underset{\otimes}{\tilde{A}}=\underset{\otimes}{\tilde{A}}$ for all $j$, then

$\operatorname{IGLOWH} A_{\omega}\left(\underset{\otimes_{1}}{\tilde{A}}, \underset{\otimes_{2}}{\tilde{A}}, \cdots, \underset{\otimes_{n}}{\tilde{A}}\right)=\underset{\otimes}{\tilde{A}}$. 
3) Theorem 3 (Monotonicity).

If $\underset{\otimes j}{\tilde{A}} \leq \underset{\otimes j}{\tilde{A}}$ for all $j$, then

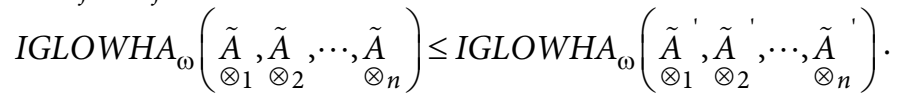

The IGLWHA operator only focuses on the importance of each interval grey linguistic variable itself, and IGLOWHA operator only weights the position of each interval grey linguistic variable, therefore, both of them have certain one-sidedness. In order to overcome the above weaknesses, the interval grey linguistic variable hybrid weighted harmonic aggregation operator is defined as follows:

Definition 11: Let $\underset{\otimes_{j}}{\tilde{A}}=\left(s_{\alpha_{j}},\left[g_{j}^{L}, g_{j}^{U}\right]\right)$ be the group of interval grey linguistic variables, and IGLHWHA: $\Omega^{n} \rightarrow \Omega$, if

$$
\operatorname{IGLHWHA} \underset{\omega, \tilde{\otimes}}{ }\left(\underset{\otimes_{1}}{\tilde{A}}, \underset{\otimes_{2}}{\tilde{A}}, \cdots, \underset{\otimes_{n}}{\tilde{A}}\right)=\left(\sum_{j=1}^{n} \frac{\omega_{j}}{\tilde{B}}\right)^{-1},
$$

where $\Omega$ is the set of all the interval grey linguistic variables, $\omega=\left(\omega_{1}, \omega_{2}, \cdots, \omega_{n}\right)$ is the weight vector associated with the function IGLHWHA, and $\sum_{j=1}^{n} \omega_{j}=1 . \underset{\otimes}{\tilde{W}}=\left(\begin{array}{l}\tilde{W} \\ \otimes_{1}\end{array} \underset{\otimes_{2}}{\tilde{W}}, \cdots, \underset{\otimes_{n}}{\tilde{W}}\right)$ is the weight vector of $\underset{\otimes_{j}}{\tilde{A}}(j=1,2, \cdots, n)$, and $\underset{\otimes_{j}}{\tilde{W}}$ is the interval grey linguistic variable $\underset{\tilde{A}}{\operatorname{represented}} \underset{\otimes_{j}}{\tilde{W}}=\left(s_{w_{j}},\left[w_{j}^{L}, w_{j}^{U}\right]\right) \cdot\left(\sigma_{1}, \sigma_{2}, \cdots, \sigma_{n}\right)$ is a permutation of $(1,2, \cdots, n)$, and $\underset{\otimes_{j}}{\tilde{B}}=\frac{\tilde{A}}{\underset{\otimes_{j}}{\tilde{W}}}=\left(s_{\beta_{j}},\left[g_{B_{j}}^{L}, g_{B_{j}}^{U}\right]\right)$, for any $j$, we can get $\underset{\otimes_{\sigma_{j-1}}}{\tilde{B}} \underset{\otimes_{\sigma_{j}}}{\tilde{B}}$, then IGLHWHA is called the interval grey linguistic hybrid weighted harmonic aggregation ( IGLHWHA )operator.

Example 3. Assume $\omega=\left(\frac{1}{8}, \frac{3}{8}, \frac{3}{8}, \frac{1}{8}\right)$ (calculated by Eq. 23) and

$$
\begin{aligned}
& \underset{\otimes_{1}}{\tilde{A}}=\left(s_{2},[0.2,0.4]\right), \underset{\otimes_{2}}{\tilde{A}}=\left(s_{1},[0.1,0.3]\right), \underset{\otimes_{3}}{\tilde{A}}=\left(s_{5},[0.4,0.5]\right), \underset{\otimes_{4}}{\tilde{A}}=\left(s_{4},[0.3,0.6]\right) ; \\
& \underset{\otimes_{1}}{\tilde{W}}=\left(s_{3},[0.3,0.4]\right), \underset{\otimes_{2}}{\tilde{W}}=\left(s_{4},[0.4,0.4]\right), \underset{\otimes_{3}}{\tilde{W}}=\left(s_{2},[0.5,0.7]\right), \underset{\otimes_{4}}{\tilde{W}}=\left(s_{4},[0.2,0.6]\right) .
\end{aligned}
$$

Firstly, calculate the $\underset{\otimes_{j}}{\tilde{B}}=\frac{\tilde{A}}{\otimes_{j}}$.

$$
\underset{\otimes_{1}}{\tilde{B}}=\frac{\underset{A}{\otimes}}{\underset{\otimes_{1}}{\tilde{W}}}=\frac{\left(s_{2},[0.2,0.4]\right)}{\left(s_{3},[0.3,0.4]\right)}=\left(s_{2 / 3},[\max (0.2,0.3), \max (0.4,0.4)]\right)=\left(s_{0.67},[0.3,0.4]\right) ;
$$




$$
\begin{aligned}
& \underset{\otimes_{2}}{\tilde{B}}=\frac{\underset{\otimes_{2}}{\tilde{A}}}{\tilde{\bigotimes}_{2}}=\frac{\left(s_{1},[0.1,0.3]\right)}{\left(s_{4},[0.4,0.4]\right)}=\left(s_{1 / 4},[\max (0.1,0.4), \max (0.3,0.4)]\right)=\left(s_{0.25},[0.4,0.4]\right) ; \\
& \underset{\otimes_{3}}{\tilde{B}}=\frac{\underset{\otimes_{3}}{\tilde{A}}}{{\underset{\bigotimes}{\tilde{W}}}_{3}}=\frac{\left(s_{5},[0.4,0.5]\right)}{\left(s_{2},[0.5,0.7]\right)}=\left(s_{5 / 2},[\max (0.4,0.5), \max (0.5,0.7)]\right)=\left(s_{2.50},[0.5,0.7]\right) ; \\
& \underset{\otimes_{4}}{\tilde{B}}=\frac{\underset{\otimes}{\otimes_{4}}}{\tilde{W}_{\otimes}}=\frac{\left(s_{4},[0.3,0.6]\right)}{\left(s_{4},[0.2,0.6]\right)}=\left(s_{4 / 4},[\max (0.3,0.2), \max (0.6,0.6)]\right)=\left(s_{1},[0.3,0.6]\right) .
\end{aligned}
$$

Secondly, calculate the expectation value of interval grey linguistic variables $\tilde{B}, \tilde{B}, \tilde{B}$ and $\tilde{B}$ according to Eq. (17).

$$
\begin{aligned}
& \otimes_{1} \otimes_{2} \otimes_{3} \otimes_{4} \\
& \text { Suppose } \rho(y)=y^{2} \text {, then } f_{\rho}([a, b])=\frac{b+2 a}{3} \text {. So, } \\
& I\left(\underset{\otimes}{\otimes_{1}}\right)=s_{0.67} \times f_{\rho}([(1-0.4),(1-0.3)])=s_{0.67} \times \frac{0.7+2 \times 0.6}{3}=s_{0.42} ; \\
& I\left(\underset{\otimes_{2}}{\tilde{B}}\right)=s_{0.25} \times f_{\rho}([(1-0.4),(1-0.4)])=s_{0.25} \times \frac{0.6+2 \times 0.6}{3}=s_{0.15} ; \\
& I\left(\underset{\otimes_{3}}{\tilde{B}}\right)=s_{2.5} \times f_{\rho}([(1-0.7),(1-0.5)])=s_{2.5} \times \frac{0.5+2 \times 0.3}{3}=s_{0.92} ; \\
& I\left(\tilde{B}_{4}\right)=s_{1} \times f_{\rho}([(1-0.6),(1-0.3)])=s_{1} \times \frac{0.7+2 \times 0.4}{3}=s_{0.5} .
\end{aligned}
$$

Thirdly, we rank the $\tilde{B}, \tilde{B}, \tilde{B}$ and $\tilde{B}$, the results are shown as follows:

$$
\begin{gathered}
\otimes_{1} \otimes_{2} \otimes_{3} \otimes_{4} \\
\sigma_{1}=3, \sigma_{2}=4, \sigma_{3}=1, \sigma_{4}=2 .
\end{gathered}
$$

Finally, By Definition 9, we have

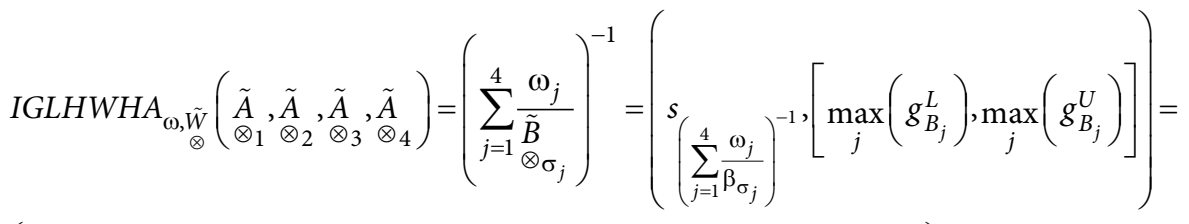

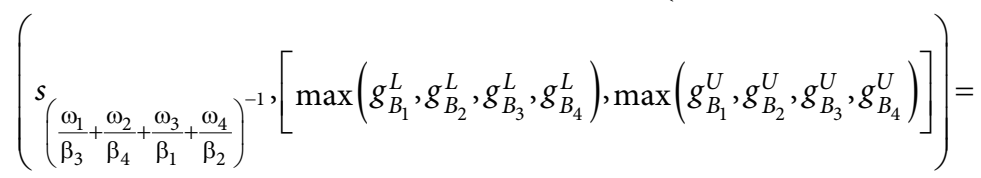

$$
\begin{aligned}
& \left(s\left(\frac{0.125}{2.5}+\frac{0.375}{1}+\frac{0.375}{0.67}+\frac{0.125}{0.25}\right)^{-1},[\max (0.3,0.4,0.5,0.3), \max (0.4,0.4,0.7,0.6)]\right)= \\
& \left(s_{(1.48)^{-1}},[0.5,0.7]\right)=\left(s_{0.67},[0.5,0.7]\right) \text {. }
\end{aligned}
$$


Obviously, IGLWHA operator and IGLOWHA operator are the special cases of the IGLHWHA operator, which not only shows the importance of interval grey linguistic variables themselves, but also shows the importance of the position of the interval grey linguistic variables.

\section{The multi-attribute group decision making method based on the IGLHWHA operator}

\subsection{The description of multiple attribute group decision making problem based on the interval grey linguistic variables}

Let $E=\left\{e_{1}, e_{2}, \cdots, e_{p}\right\}$ be the experts set in the group decision making, $A=\left\{A_{1}, A_{2}, \cdots, A_{m}\right\}$ be the set of alternatives, and $C=\left\{C_{1}, C_{2}, \cdots, C_{n}\right\}$ be the attribute set with respect to the alternatives. Supposed that $\underset{\otimes_{i j}}{\tilde{A}^{k}}=\left(t_{i j}^{k},\left[g_{i j k}^{L}, g_{i j k}^{U}\right]\right)$ is the attribute value in the attribute $C_{j}$ with respect to the alternative $A_{i}$, given by expert $e_{k}$, and $\underset{\otimes}{\tilde{A}^{k}}=\left[\begin{array}{c}\tilde{A}^{k} \\ \otimes_{i j}\end{array}\right]_{m \times n}$ is the decision making matrix given by the expert $e_{k}$, and $\underset{\otimes}{\tilde{W}^{k}}=\left(\begin{array}{c}\tilde{W}^{k}, \tilde{W}^{k} \\ {\underset{\otimes}{2}}_{2}\end{array}, \cdots, \underset{\otimes_{n}}{\tilde{W}^{k}}\right), \underset{\otimes_{j}}{\tilde{W}^{k}}=\left(\eta_{j k},\left[w_{j k}^{L}, w_{j k}^{U}\right]\right)$ is the attribute weight, where $t_{i j}^{k}, \eta_{j k} \in S, S$ is the linguistic label. Let $\lambda=\left(\lambda_{1}, \lambda_{2}, \cdots, \lambda_{p}\right)$ be the experts weight, and $\sum_{k=1}^{p} \lambda_{k}=1$. The attribute weight is unknown. We can rank the order of the alternatives based on the given information.

\subsection{Decision making steps}

1) Aggregate the evaluation information of each expert

According to the different attributes' attribute values and weights which were given by different experts under different alternatives, we can aggregate the attribute values and weights into group decision making information. Based on the IGLWHA $A_{\lambda}$ operator, we can get the group decision making matrix $\underset{\otimes}{\tilde{X}}=\left[\begin{array}{c}\tilde{X} \\ \otimes_{i j}\end{array}\right]_{m \times n}$ and the group decision making weight $\operatorname{vector} \underset{\otimes}{\tilde{W}}=\left(\underset{\otimes_{1}}{\tilde{W}}, \underset{\otimes_{2}}{\tilde{W}}, \cdots, \underset{\otimes_{n}}{\tilde{W}}\right)$, where $\underset{\otimes_{i j}}{\tilde{X}}=\left(t_{i j},\left[g_{i j}^{L}, g_{i j}^{U}\right]\right)$ and $\underset{\otimes_{j}}{\tilde{W}}=\left(\eta_{j},\left[w_{j}^{L}, w_{j}^{U}\right]\right)$. Then

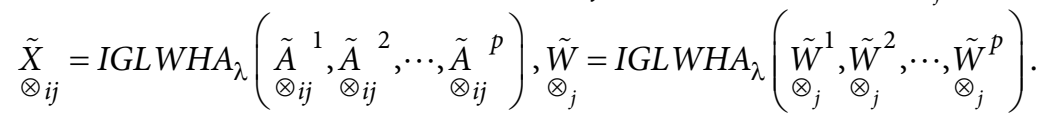


2) Calculate the comprehensive evaluation value of each alternative

We utilize the IGLHWHA operator to calculate the comprehensive evaluation value of each alternative $\underset{\otimes_{i}}{\tilde{Z}}=\left(c_{i},\left[z_{i}^{L}, z_{i}^{U}\right]\right)=I G L H W H A_{\omega, \tilde{W}}\left(\underset{\otimes_{i 1}}{\tilde{X}}, \underset{\otimes_{i 2}}{\tilde{X}}, \cdots, \underset{\bigotimes_{i n}}{\tilde{X}}\right)=\left(\sum_{j=1}^{n} \frac{\omega_{j}}{\tilde{B}}\right)^{-1}$, where $\omega=\left(\omega_{1}, \omega_{2}, \cdots, \omega_{n}\right)$ is the weight vector associated with the function, and $\sum_{j=1}^{n} \omega_{j}=1$; $\underset{\otimes}{\tilde{W}}=\left(\begin{array}{c}\tilde{W} \\ \otimes_{1}\end{array} \underset{\otimes_{2}}{\tilde{W}}, \cdots, \underset{\otimes_{n}}{\tilde{W}}\right)$ is the weight vector of $\underset{\otimes_{i j}}{\tilde{X}}(j=1,2, \cdots, n),\left(\sigma_{1}, \sigma_{2}, \cdots, \sigma_{n}\right)$ is a permuta-

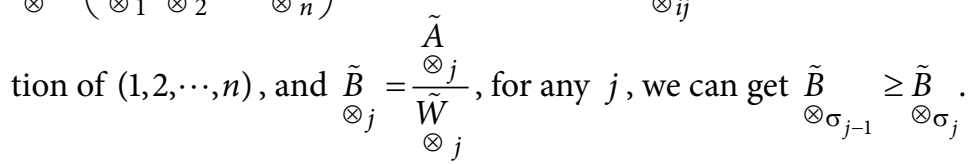

3) Rank the alternatives

Because $\tilde{Z}$ is the interval grey linguistic variables, we can get the ranking alternatives by the expectation value $I(\tilde{Z})$. The larger the value $\left.\underset{\otimes_{i}}{I\left(\tilde{Z}_{i}\right.}\right)$ is, the better the alternative is.

The flowchart of this method is shown in Fig. 1.

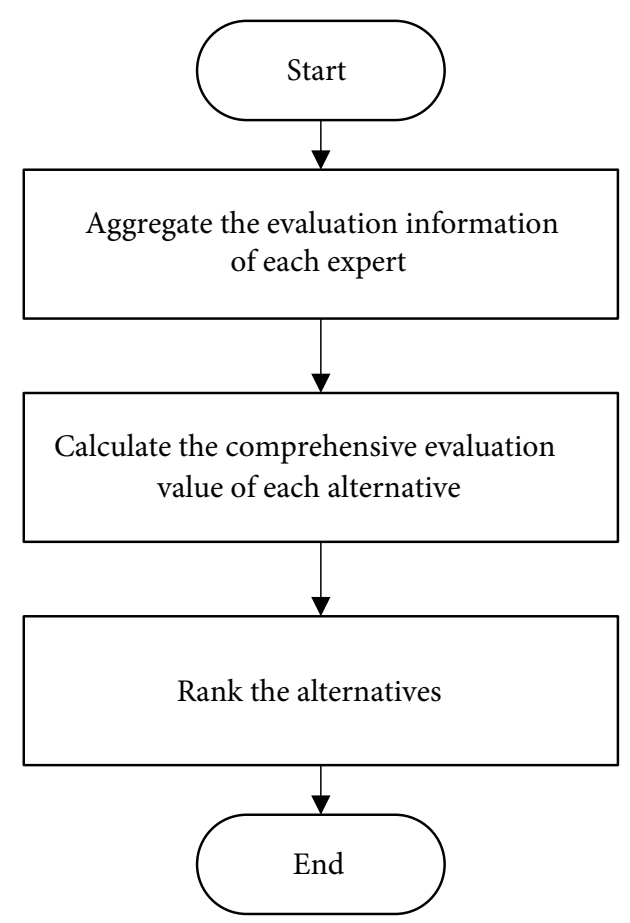

Fig. 1. The flowchart of the proposed method 


\section{Practical examples}

To evaluate the performance of the proposed method, practical examples are presented as follows: now there are four enterprises $\left\{A_{1}, A_{2}, A_{3}, A_{4}\right\}$, the object is to evaluate the technological innovation ability of the enterprises.

The first step is to develop the evaluation criterion for the project. The criterion is shown as follows: the ability of innovative resources investment $\left(C_{1}\right)$, the ability of innovation management $\left(C_{2}\right)$, the ability of innovation tendency $\left(C_{3}\right)$ and the ability of research and development $\left(C_{4}\right)$. Based on the four criterions, the three experts $\left\{e_{1}, e_{2}, e_{3}\right\}$ are invited to evaluate the technological innovation ability of the four enterprises. Supposed that $\lambda=(0.4,0.32,0.28)$ be the weight vector about the three experts, and the evaluating values given by the experts adopting interval grey linguistic variables are shown in Tables 1, 2 and 3, and the criterion weight values are shown in Table 4 . Let $S=\left(s_{1}, s_{2}, s_{3}, s_{4}, s_{5}, s_{6}, s_{7}\right)$ be the linguistic label. The problem is to rank the four enterprises based on their technological innovation ability.

Table 1 . The attribute values of each attribute with respect to four enterprises given by expert $e_{1}$

\begin{tabular}{ccccc}
\hline Enterprises & Attribute $\left(C_{1}\right)$ & Attribute $\left(C_{2}\right)$ & Attribute $\left(C_{3}\right)$ & Attribute $\left(C_{4}\right)$ \\
\hline A1 & $\left(s_{5},[0.2,0.3]\right)$ & $\left(s_{2},[0.4,0.4]\right)$ & $\left(s_{5},[0.5,0.5]\right)$ & $\left(s_{3},[0.2,0.4]\right)$ \\
\hline A2 & $\left(s_{4},[0.4,0.4]\right)$ & $\left(s_{5},[0.4,0.5]\right)$ & $\left(s_{3},[0.1,0.2]\right)$ & $\left(s_{4},[0.5,0.5]\right)$ \\
\hline A3 & $\left(s_{3},[0.2,0.3]\right)$ & $\left(s_{4},[0.2,0.3]\right)$ & $\left(s_{4},[0.3,0.3]\right)$ & $\left(s_{5},[0.2,0.3]\right)$ \\
\hline A4 & $\left(s_{6},[0.5,0.6]\right)$ & $\left(s_{2},[0.2,0.2]\right)$ & $\left(s_{3},[0.2,0.4]\right)$ & $\left(s_{3},[0.3,0.4]\right)$ \\
\hline
\end{tabular}

Table 2. The attribute values of each attribute with respect to four enterprises given by expert $e_{2}$

\begin{tabular}{ccccc}
\hline Enterprises & Attribute $\left(C_{1}\right)$ & Attribute $\left(C_{2}\right)$ & Attribute $\left(C_{3}\right)$ & Attribute $\left(C_{4}\right)$ \\
\hline A1 & $\left(s_{4},[0.1,0.3]\right)$ & $\left(s_{3},[0.2,0.3]\right)$ & $\left(s_{3},[0.2,0.2]\right)$ & $\left(s_{6},[0.4,0.5]\right)$ \\
\hline A2 & $\left(s_{5},[0.4,0.5]\right)$ & $\left(s_{3},[0.3,0.4]\right)$ & $\left(s_{4},[0.2,0.4]\right)$ & $\left(s_{3},[0.2,0.3]\right)$ \\
\hline A3 & $\left(s_{4},[0.2,0.4]\right)$ & $\left(s_{4},[0.2,0.3]\right)$ & $\left(s_{2},[0.4,0.4]\right)$ & $\left(s_{3},[0.3,0.3]\right)$ \\
\hline A4 & $\left(s_{5},[0.3,0.4]\right)$ & $\left(s_{4},[0.4,0.5]\right)$ & $\left(s_{2},[0.3,0.4]\right)$ & $\left(s_{4},[0.2,0.4]\right)$ \\
\hline
\end{tabular}

Table 3. The attribute values of each attribute with respect to four enterprises given by expert $e_{3}$

\begin{tabular}{ccccc}
\hline Enterprises & Attribute $\left(C_{1}\right)$ & Attribute $\left(C_{2}\right)$ & Attribute $\left(C_{3}\right)$ & Attribute $\left(C_{4}\right)$ \\
\hline A1 & $\left(s_{5},[0.2,0.4]\right)$ & $\left(s_{3},[0.3,0.3]\right)$ & $\left(s_{4},[0.4,0.5]\right)$ & $\left(s_{4},[0.2,0.3]\right)$ \\
\hline A2 & $\left(s_{4},[0.3,0.3]\right)$ & $\left(s_{5},[0.3,0.4]\right)$ & $\left(s_{2},[0.1,0.2]\right)$ & $\left(s_{3},[0.1,0.2]\right)$ \\
\hline A3 & $\left(s_{4},[0.2,0.3]\right)$ & $\left(s_{5},[0.3,0.4]\right)$ & $\left(s_{1},[0.1,0.2]\right)$ & $\left(s_{4},[0.2,0.3]\right)$ \\
\hline A4 & $\left(s_{3},[0.2,0.3]\right)$ & $\left(s_{3},[0.1,0.3]\right)$ & $\left(s_{4},[0.3,0.4]\right)$ & $\left(s_{5},[0.4,0.5]\right)$ \\
\hline
\end{tabular}


Table 4 . The attribute weight value given by experts

\begin{tabular}{ccccc}
\hline Experts & Attribute $\left(C_{1}\right)$ & Attribute $\left(C_{2}\right)$ & Attribute $\left(C_{3}\right)$ & Attribute $\left(C_{4}\right)$ \\
\hline$e_{1}$ & $\left(s_{5},[0.2,0.3]\right)$ & $\left(s_{3},[0.1,0.2]\right)$ & $\left(s_{2},[0.3,0.4]\right)$ & $\left(s_{3},[0.2,0.3]\right)$ \\
\hline$e_{2}$ & $\left(s_{3},[0.2,0.3]\right)$ & $\left(s_{4},[0.2,0.4]\right)$ & $\left(s_{3},[0.1,0.2]\right)$ & $\left(s_{3},[0.1,0.2]\right)$ \\
\hline$e_{3}$ & $\left(s_{4},[0.2,0.2]\right)$ & $\left(s_{3},[0.1,0.2]\right)$ & $\left(s_{2},[0.1,0.2]\right)$ & $\left(s_{3},[0.2,0.3]\right)$ \\
\hline
\end{tabular}

The evaluation steps used in this paper are proposed as follows:

(1) Based on the Eq. (19), to aggregate the evaluation information (shown in Tables 1, 2, 3 and 4$)$ about the experts $\left\{e_{1}, e_{2}, e_{3}\right\}$, then we can get the group decision making matrix $\underset{\otimes}{\tilde{X}}$ and attribute weight vector $\underset{\otimes}{\tilde{W}}$ :

$$
\begin{aligned}
& \tilde{X}=\left[\begin{array}{cccc}
\left(s_{4.63},[0.20,0.40]\right) & \left(s_{2.50},[0.40,0.40]\right) & \left(s_{3.90},[0.50,0.50]\right) & \left(s_{3.90},[0.40,0.50]\right) \\
\left(s_{4.27},[0.40,0.50]\right) & \left(s_{4.12},[0.40,0.50]\right) & \left(s_{2.83},[0.20,0.40]\right) & \left(s_{3.33},[0.50,0.50]\right) \\
\left(s_{3.53},[0.20,0.40]\right) & \left(s_{4.24},[0.30,0.40]\right) & \left(s_{1.85},[0.40,0.40]\right) & \left(s_{3.90},[0.30,0.30]\right) \\
\left(s_{4.46},[0.50,0.60]\right) & \left(s_{2.68},[0.40,0.50]\right) & \left(s_{2.75},[0.30,0.40]\right) & \left(s_{3.71},[0.40,0.50]\right)
\end{array}\right] ; \\
& \underset{\otimes}{\tilde{W}}=\left(\left(s_{3.90},[0.20,0.30]\right),\left(s_{3.26},[0.20,0.40]\right),\left(s_{2.24},[0.30,0.40]\right),\left(s_{3.00},[0.20,0.30]\right)\right) .
\end{aligned}
$$

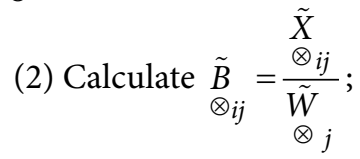

$$
\underset{B}{\otimes}=\left[\begin{array}{c}
\left(s_{1.188},[0.20,0.40]\right),\left(s_{0.767},[0.40,0.40]\right),\left(s_{1.740},[0.50,0.50]\right),\left(s_{1.299},[0.40,0.50]\right) \\
\left(s_{1.097},[0.40,0.50]\right),\left(s_{1.264},[0.40,0.50]\right),\left(s_{1.264},[0.30,0.40]\right),\left(s_{1.111},[0.50,0.50]\right) \\
\left(s_{0.906},[0.20,0.40]\right),\left(s_{1.299},[0.30,0.40]\right),\left(s_{0.827},[0.40,0.40]\right),\left(s_{1.299},[0.30,0.30]\right) \\
\left(s_{1.146},[0.50,0.60]\right),\left(s_{0.821},[0.40,0.50]\right),\left(s_{1.229},[0.30,0.40]\right),\left(s_{1.238},[0.40,0.50]\right)
\end{array}\right] .
$$

3) Calculate the comprehensive evaluation values of each alternative

If the BUM function is $\rho(y)=y^{2}$, then $f_{\rho}([a, b])=\frac{b+2 a}{3}$. The position vector is determined by the equation (23), and $\omega=\left(\frac{1}{8}, \frac{3}{8}, \frac{3}{8}, \frac{1}{8}\right)$. According to the equation (24), we can get the comprehensive evaluation values of each alternative:

$$
\underset{Z}{\otimes}=\left(\begin{array}{l}
\mathrm{s}_{1.19},[0.50,0.50] \\
\mathrm{s}_{1.18},[0.50,0.50] \\
\mathrm{s}_{1.05},[0.40,0.40] \\
\mathrm{s}_{1.13},[0.50,0.60]
\end{array}\right)
$$


4) Rank the alternatives.

We select the BUM function $\rho(y)=y^{2}$; the expectation value $I\left(\underset{\otimes_{i}}{\tilde{Z}}\right)$ is calculated by Eq. (17).

$$
I\left(\underset{\otimes_{1}}{\tilde{Z}}\right)=s_{0.596}, I\left(\underset{\otimes_{2}}{\tilde{Z}}\right)=s_{0.588}, I\left(\underset{\otimes_{3}}{\tilde{Z}}\right)=s_{0.632}, I\left(\underset{\otimes_{4}}{\tilde{Z}}\right)=s_{0.490} .
$$

So the orders of technological innovation ability of the four enterprises $\left\{A_{1}, A_{2}, A_{3}, A_{4}\right\}$ are shown as follows:

$$
A_{3} \succ A_{1} \succ A_{2} \succ A_{4}
$$

5) Discuss.

In order to illustrate the effect of the position weight vector $\omega$ on decision making of this example, we use the different value $\omega$ to rank the alternatives, and the ranking results are shown as follows:

1) For $\omega=\left(\frac{1}{4}, \frac{1}{4}, \frac{1}{4}, \frac{1}{4}\right)$, the orders are $A_{3} \succ A_{2} \succ A_{1} \succ A_{4}$.

2) For $\omega=\left(\frac{2}{5}, \frac{1}{5}, \frac{1}{5}, \frac{1}{5}\right)$ which can be calculated by Eq. (22) in $\alpha=0.2$, the orders are $A_{3} \succ A_{1} \succ A_{2} \succ A_{4}$.

3) For $\omega=\left(\frac{3}{8}, \frac{1}{8}, \frac{1}{8}, \frac{3}{8}\right)$, the orders are $A_{3} \succ A_{2} \succ A_{1} \succ A_{4}$.

These show that the position weight vector $\omega$ has a certain impact on ranking.

In addition, in order to verify the validity of the method proposed in this paper, we use the method proposed by Meng et al. (2007) to sort this example. Because the method proposed by Meng et al. (2007) is for grey fuzzy decision making problems in which the fuzzy part and the grey part of grey fuzzy numbers take the form of the interval numbers, and in this paper, the fuzzy part of grey fuzzy numbers takes the form of the linguistic variables, and the grey part takes the form of the interval numbers. So in order to use the method proposed by Meng et al. (2007), we change the linguistic variables of fuzzy part of grey fuzzy numbers to interval numbers according to Liu and Meng (2009) firstly. The ranking result is shown as follows:

$$
A_{3} \succ A_{2} \succ A_{1} \succ A_{4}
$$

Obviously, two methods have the same ranking results; this verifies the validity of the method in this paper.

In order to further illustrate the validity of the proposed method, we use the evaluation data presented by Meng et al. (2007). Firstly, we convert the interval numbers to the linguistic variables by the methods proposed by Liu (2009), and then we can use the methods in this paper. By calculating, we get the ranking of 4 alternatives. It is shown as follows:

$$
y_{2} \succ y_{4} \succ y_{1} \succ y_{3}
$$

It is the same as the ranking result produced in Meng et al. (2007). 


\section{Conclusions}

The traditional grey fuzzy decision making methods are generally suitable for decision making the information taking the form of crisp numbers, or interval numbers in the fuzzy part and the grey part of grey fuzzy numbers, and yet they will fail in dealing with the linguistic information of grey fuzzy numbers. In this paper, with respect to multiple attribute group decision making (MAGDM) problems in which the attribute values and the attribute weights take the form of the interval grey linguistic variables, some new group decision making analysis methods are developed. Firstly, the concept of interval grey linguistic variables is proposed, in which the fuzzy part takes the form of the linguistic variables, and the grey part takes the form of the interval numbers. Then, the operation rules of interval grey linguistic variables are defined, and some operators (such as interval grey linguistic weighted harmonic aggregation (IGLWHA) operator, interval grey linguistic ordered weighted harmonic aggregation (IGLOWHA) operator, and interval grey linguistic hybrid weighted harmonic aggregation (IGLHWHA) operator) are proposed to solve the group decision making problems. Finally, the computational results from an illustrative example have shown that the proposed approach is feasible and effective for the group-decision making problems, and it is easier to understand and implement. This study promotes the development of the theory and method of grey fuzzy multiple attribute decision making and provides a new idea to solve the grey fuzzy multiple attribute decision making. Because interval grey linguistic variables take into account the greyness and fuzziness of the same problem, they can get more rational decision-making results. However, in order to do this, we must get more data, and sometimes, it is very difficult to obtain these data. In the future, we will research data acquisition methods, and continue working in the extension and application of the developed operators and methods to other domains.

\section{Acknowledgement}

This paper is supported by the National Natural Science Foundation of China (No. 71271124), the Humanities and Social Sciences Research Project of Ministry of Education of China (No. 10YJA630073, No. 09YJA630088), the Natural Science Foundation of Shandong Province (No. ZR2011FM036), National High Technology Research and Development Program (863 Program) Foundation (No. 2011AA100700) and Technology innovation fund project of high technology-based SMEs(2012-34-2). The author also would like to express appreciation to the anonymous reviewers and Associate Editor for their very helpful comments that improved the paper.

\section{References}

Alonso, S.; Cabrerizo, F. J.; Chiclana, F.; Herrera, F.; Herrera-Viedma, E. 2009. Group decision-making with incomplete fuzzy linguistic preference relations, International Journal of Intelligent Systems 24(2): 201-222.

Bu, G. Z.; Zhang, Y. W. 2002. Grey fuzzy comprehensive evaluation based on the theory of grey fuzzy relation, Systems Engineering Theory and Practice 22(4): 141-144. 
Cabrerizo, F. J.; Heradio, R.; Pérez, I. J.; Herrera-Viedma, E. 2010a. A selection process based on additive consistency to deal with incomplete fuzzy linguistic information, Journal of Universal Computer Science 16(1): 62-81.

Cabrerizo, F. J.; Pérez, I. J.; Herrera-Viedma, E. 2010b. Managing the consensus in group decision making in an unbalanced fuzzy linguistic context with incomplete information, Knowledge-Based Systems 23(2): 169-181.

Chen, D. W. 1994. Grey fuzzy set introduction. Harbin: Helongjiang Science and Technology Press.

Choobineh, F.; Li, H. 1993b. Ranking fuzzy multi-criteria alternatives with respect to a decision maker's fuzzy goal, Information Science 72(1-2): 143-155.

Choobineh, F.; Li, H. 1993a. An index for ordering fuzzy numbers, Fuzzy Set and Systems 54(3): 287-294.

Deng, J. L. 1982. Control problems of grey systems, Systems \& Control Letters 1(5): 288-294.

Deng, J. L. 2002. Grey Prediction and Grey Decision. Wuhan: Huazhong University Press.

Herrera, F.; Herrera-Viedma, E.; Verdegay, J. L. 1996. A model of consensus in group decision making under linguistic assessments, Fuzzy Sets and Systems 79(1): 73-87.

Herrera, F.; Alonso, S.; Chiclana, F.; Herrera-Viedma, E. 2009. Computing with words in decision making: foundations, trends and prospects, Fuzzy Optimization and Decision Making 8(4): 337-364.

Herrera, F.; Herrera-Viedma, E. 2000. Linguistic decision analysis: steps for solving decision problems under linguistic information, Fuzzy Sets and Systems 115(1): 67-82.

Herrera-Viedma, E.; Cordón, O.; Luque, M.; Lopez, A. G. 2003. A model of fuzzy linguistic IRS based on multi-granular linguistic information, International Journal of Approximate Reasoning 34(2-3): 221-239.

Jin, N.; Lou, S. C. 2003. Study on multi-attribute decision making model based on the theory of grey fuzzy relation, Intelligence Command Control and Simulation Techniques 7: 44-47.

Jin, N.; Lou, S. C. 2004. A grey fuzzy multi-attribute decision making method, Fire Control \& Command Control 29(4): 26-28.

Kim, S. H.; Ahn, B. S. 1999. Interactive group decision making procedure under incomplete information, European Journal of Operational Research 116: 498-507. http://dx.doi.org/10.1016/S0377-2217(98)00040-X

Li, H. X.; Wang, P. Z. 1994. Fuzzy mathematical. Beijing: National Defense Industry Press.

Liu, P. D. 2009. A novel method for hybrid multiple attribute decision making, Knowledge-Based Systems 22(5): 388-391. http://dx.doi.org/10.1016/j.knosys.2009.02.001

Liu, P. D. 2011. A weighted aggregation operators multi-attribute group decision-making method based on interval-valued trapezoidal fuzzy numbers, Expert Systems with Applications 38(1): 1053-1060. http://dx.doi.org/10.1016/j.eswa.2010.07.144

Liu, P. D.; Meng, F. K. 2009. The research of supplier selection based on hybrid decision-making index and projection method, Journal of Computational Information System 5(2): 601-610.

Liu, P. D.; Zhang, X. 2010. The study on multi-attribute decision-making with risk based on linguistic variable, International Journal of Computational Intelligence Systems 3(5): 601-609. http://dx.doi.org/10.1080/18756891.2010.9727726

Liu, P. D; Su, Y. 2010. The multiple attribute decision making method based on the TFLHOWA operator, Computers and Mathematics with Applications 60(9): 2609-2615. http://dx.doi.org/10.1016/j.camwa.2010.08.087

Liu, W. L.; Liu, P. D. 2010. Hybrid multiple attribute decision making method based on relative approach degree of grey relation projection, African Journal of Business Management 4(17): 3716-3724.

$\mathrm{Lu}, \mathrm{R} .2009$. Study on the analysis methods of grey fuzzy multi-attribute decision making. Master's thesis. Zhejiang Gongshang University. 
Luo, D. 2005. The analysis methods on grey decision-making problems. Zhengzhou: Yellow River and Water Conservancy Press.

Luo, D.; Liu, S. 2004. Analytic method to a kind of grey fuzzy decision making based on entropy, Engineering Science 6(10): 48-51.

Martínez, L.; Ruan, D.; Herrera, F.; Herrera-Viedma, E.; Wang, P. P. 2009. Linguistic decision making: Tools and applications, Information Sciences 179(14): 2297-2298. http://dx.doi.org/10.1016/j.ins.2008.12.017

Meng, K.; Li, Y. X.; Wang, C. J.; Yang, W. T. 2007. Interval-value grey fuzzy comprehensive evaluation based on the preference of the risk and its application, Fire Control and Command Control 32(4): 109-111.

Razavi Hajiagha, S. H.; Hashemi, S. S.; Zavadskas, E. K. 2013. A complex proportional assessment method for group decision making in an interval-valued intuitionistic fuzzy environment, Technological and Economic Development of Economy 19(1): 22-37. http://dx.doi.org/10.3846/20294913.2012.762953

Wang, C. H.; Song, L. T. 1988. Fuzzy theory and methodology. Beijing: China Building Industry Press.

Wang, J. Q.; Wang, J. 2008. Interval grey fuzzy multi-criteria decision making approach, Systems Engineering and Electronics 30(12): 2409-2411.

Wang, Q. Y. 1996. Grey fuzzy mathematical foundation. Wuhan: Huazhong University of Science and Technology Press.

Wang, Y.; Xu, Z. S. 2008. A new method of giving OWA weights, Mathematics in Practice and Theory 38(3): 51-61.

$\mathrm{Xu}, \mathrm{Z}$. S. 2004. EOWA and EOWG operators for aggregating linguistic labels based on linguistic preference relations, International Journal of Uncertainty, Fuzziness and Knowledge-Based Systems 12: 791-810. http://dx.doi.org/10.1142/S0218488504003211

$\mathrm{Xu}, \mathrm{Z}$. S. 2006a. A note on linguistic hybrid arithmetic averaging operator in multiple attribute group decision making with linguistic information, Group Decision and Negotiation 15(6): 593-604. http://dx.doi.org/10.1007/s10726-005-9008-4

Xu, Z. S. 2006b. Goal programming models for multiple attribute decision making under linguistic setting, Journal of Management Sciences in China 9(2): 9-17.

$\mathrm{Xu}, \mathrm{Z}$. S. 2007. A method for multiple attribute decision making with incomplete weight information in linguistic setting, Knowledge-Based Systems 20(8): 719-725. http://dx.doi.org/10.1016/j.knosys.2006.10.002

$\mathrm{Xu}, \mathrm{Z}$. S. 2008. Group decision making based on multiple types of linguistic preference relations, Information Sciences 178(2): 452-467. http://dx.doi.org/10.1016/j.ins.2007.05.018

Yager, R. R. 2004. OWA aggregation over a continuous interval argument with applications to decision making, IEEE Transactions on Systems, Man, and Cybernetics. Part B, Cybernetics 34(5): 1952-1963. http://dx.doi.org/10.1109/TSMCB.2004.831154

Yu, D. J. 2013. Intuitionistic fuzzy prioritized operators and their application in multi-criteria group decision making, Technological and Economic Development of Economy 19(1): 1-21. http://dx.doi.org/10.3846/20294913.2012.762951

Zadeh, L. A. 1965. Fuzzy sets, Information and Control 8: 338-356. http://dx.doi.org/10.1016/S0019-9958(65)90241-X

Zhang, X.; Liu, P. D. 2010. Method for multiple attribute decision-making under risk with interval numbers, International Journal of Fuzzy Systems 12(3): 237-242.

Zhang, N. 2013. Method for aggregating correlated interval grey linguistic variables and its application to decision making, Technological and Economic Development of Economy 19(2): 189-202. http://dx.doi.org/10.3846/20294913.2012.763071

Zhu, S. Q.; Meng, K.; Zhang, H. X. 2006. Interval numbers grey fuzzy comprehensive evaluation and its application, Electronics Optics and Control 13(3): 36-37, 41. 
Fang JIN has a PhD and is a chief research worker at The Mathematical and Economic Research Institute in Shandong University of Finance and Economics, China. She is the author of more than 30 research papers. Her research interests include decision-making theory, expert systems, and their applications.

Peide LIU has a $\mathrm{PhD}$ and is a chief research worker at School of Management Science and Engineering, Shandong University of Finance and Economics, China. He has authored or coauthored more than 90 publications. His research interests include aggregation operators, fuzzy logics, fuzzy decision making, and their applications.

Xin ZHANG has a PhD and is a Director of the School of Management Science and Engineering at The Mathematical and Economic Research Institute at Shandong University of Finance and Economics, China. He is the author of more than 50 research papers. His research interests include decision-making theory, expert systems, and their applications. 